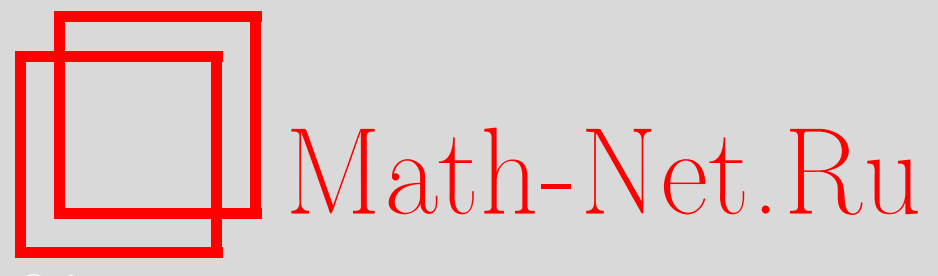

А. В. Чашкин, Моделирование схем из функциональных элементов на универсальной машине Тьюринга, Дискрет. матем., 2004, том 16, выпуск 2, 98-103

DOI: https://doi.org/10.4213/dm155

Использование Общероссийского математического портала Math-Net.Ru подразумевает, что вы прочитали и согласны с пользовательским соглашением http://www . mathnet.ru/rus/agreement

Параметры загрузки:

IP : 35.173 .219 .12

26 апреля 2023 г., 14:02:08

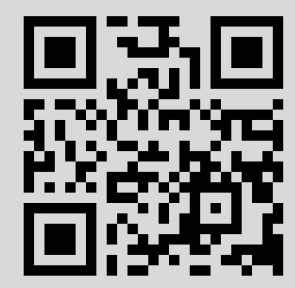


Удк 519.7

\title{
Моделирование схем из функциональных элементов на универсальной машине Тьюринга
}

๑) 2004 г. А. В. Чашкин

\begin{abstract}
Рассматривается время моделирования булевых схем на машине Тьюринга с тремя лентами, одна из которых используется для хранения программы, управляющей работой машины. Установлено, что для любой схемы $S$ найдется такая программа $P$, что время моделирования $T(P)$ схемы $S$ удовлетворяет соотношению $T(P)=$ $O\left(L(S) \log _{2} L(S)\right)$, где $L(S)$ - сложность схемы $S$. Показано, что для некоторых схем данное соотношение является точным, то есть для них существуют нижние оценки $T(P)$ того же порядка.

Работа выполнена при поддержке Российского фонда фундаментальных исследований, проекты 02-01-00985 и 00-15-96103.
\end{abstract}

1. Значительный интерес представляет задача сравнения вычислительной мощности различных моделей вычислений, в частности, вычислений, использующих условный переход, и вычислений, условный переход не использующих. Типичными представителями вычислений первого типа являются различные варианты многоленточных машин Тьюринга. Описания машин Тьюринга и оценки времени моделирования одних машин Тьюринга другими можно найти в [1-8]. Основными моделями вычислений без условного перехода являются схемы (схемы из функциональных элементов, булевы схемы) и неветвящиеся программы. Описания и свойства этих моделей можно найти в $[1,3]$. Отметим, что несмотря на внешнее несходство определений схем и неветвящихся программ (в основе определения схемы лежит понятие графа, а неветвящиеся программы определяются как последовательности равенств), схемы и неветвящиеся программы являются практически одним и тем же объектом. Поэтому результаты о сложности схем почти дословно можно переносить на сложность неветвящихся программ и наоборот.

Оценки сложности схем, моделирующих работу машины Тьюринга, можно найти в [3, $8]$, где, в частности, показано, что $T$ шагов машины Тьюринга можно промоделировать схемой, сложность которой по порядку не превосходит величины $T \log _{2} T$. Если же во время работы машина Тьюринга использует не более $m$ различных ячеек, то сложность моделирующей схемы по порядку не превосходит величины $T \log _{2} m$. Связь длины программ универсальных машин Тьюринга и схемной сложности подробно рассматривается в [3], где под универсальной машиной Тьюринга понимается машина, способная моделировать, при подходящей программе, работу произвольной машины Тьюринга.

В настоящей работе изучается время моделирования булевых схем (схем, вычисляющих булевы функщии) на универсальной трехленточной машине Тьюринга. В ряде работ (см., например, $[3,8,9])$ рассматривались похожие задачи. 
2. Приведем необходимые определения. Понятия, используемые ниже без определений, можно найти в [1-3].

Машиной Тьюринга $M$ будем называть конечный автомат $Q$, снабженный тремя двухсторонними лентами $A, B$ и $C$, ячейки которых пронумерованы целыми числами так, что ячейки с меньшими номерами находятся левее ячеек с бо́льшими номерами. В каждый момент времени автомат имеет доступ к одной (активной) ячейке каждой ленты и в зависимости от величин, содержащихся в активных ячейках лент, и своего внутреннего состояния может

- изменить содержимое активных ячеек на лентах $A$ и $B$;

- сдвинуть каждую из лент на одну ячейку либо вправо, либо влево;

- изменить внутреннее состояние автомата $Q$.

Ленты $A$ и $B$ называются рабочими, используются для записи промежуточных результатов вычислений и в каждый момент времени либо пусты, либо содержат только булевы величины. Лента $C$ называется программной. Ее алфавит состоит из конечного числа элементов $c_{1}, \ldots, c_{k}$, которые будем называть командами. Последовательность команд, находящаяся на ленте $C$, называется программой, управляюшей работой машины. Далее рассматриваются только безусловные программы, на каждом шаге такой программы автомат $Q$ выполняет команду, содержащуюся в активной ячейке ленты $C$, после чего сдвигает ленту $C$ влево. Будем полагать, что число команд $k$ и число внутренних состояний автомата $Q$ достаточно велики для того, чтобы машина $M$ могла выполнять описываемые далее действия.

Булевой схемой $S$ с $n$ входами над множеством независимых переменных $\left\{x_{1}, \ldots, x_{n}\right\}$ будем называть последовательность присваиваний

$$
y_{i}=f_{i}\left(a_{i}, b_{i}\right), \quad i=1,2, \ldots, k,
$$

где $f_{i}$ - двухместная булева функция, $a_{i}, b_{i} \in\left\{x_{1}, \ldots, x_{n}, y_{1}, \ldots, y_{i-1}\right\}$. Величины $y_{1}, y_{2}, \ldots$ называются внутренними переменными схемы, а равенства $(1)$ - элементами схемы. Число равенств называется сложностью схемы и обозначается через $L(S)$. Функции, вычисляемые схемами, сложность вычисления функций схемами и минимальные схемы определяются как обычно (см., например, [1, 3]).

Пусть схема $S$ вычисляет систему булевых функций

$$
f_{1}\left(x_{1}, \ldots, x_{n}\right), \ldots, f_{m}\left(x_{1}, \ldots, x_{n}\right)
$$

Будем говорить, что машина $M$ под управлением программы $P$ моделирует работу $S$, если выполняются следующие два условия.

(1) В начальный момент времени значения переменных $x_{1}, \ldots, x_{n}$ последовательно (в соседних ячейках) расположены на ленте $A$. Команды программы $P$ расположены на ленте $C$. На лентах $A$ и $C$ активными являются самые левые непустые ячейки. Лента $B$ пуста.

(2) После окончания работы машины значения $f_{1}\left(x_{1}, \ldots, x_{n}\right), \ldots, f_{m}\left(x_{1}, \ldots, x_{n}\right)$ (возможно в ином порядке) последовательно расположены на ленте $A$. На ленте $A$ активной является самая левая непустая ячейка. Лента $B$ пуста. 
3. Рассмотрим список $l=\left(l_{1}, \ldots, l_{n}\right)$ и двоичный набор $\alpha=\left(\alpha_{1}, \ldots, \alpha_{n}\right)$. Список $l(\alpha)=\left(l_{i_{1}}, \ldots, l_{i_{n}}\right)$ назовем $\alpha$-перестановкой списка $l$, если $\alpha_{i_{j}} \leqslant \alpha_{i_{j+1}}$ для каждого $j=1,2, \ldots, n-1$. Будем говорить, что машина Тьюринга $M$ выполняет $\alpha$-перестановку $n$-элементного списка $l$, если перед началом работы элементы списка $l$ последовательно расположены на ленте $A$ и самая левая непустая ячейка ленты $A$ является активной; во время работы машины ни одна из ячеек, расположенных на ленте $A$ правее ячеек с элементами списка, не является активной; после окончания работы элементы списка $l(\alpha)$ последовательно расположены на ленте $A$, и самая левая непустая ячейка ленты $A$ является активной.

Лемма 1. Произвольную $\alpha$-перестановку $n$-элементного списка l можно выполнить на машине Тьюринга $M$ за время, не превосходящее $6 n$.

Доказательство. Без ограничения общности будем полагать, что элементы списка находятся на ленте $A$ в ячейках с номерами от 1 до $n$, а на ленте $B$ активной является ячейка с номером 1. Последовательно перенесем элементы списка с ленты $A$ на ленту $B$. Элемент, находящийся на ленте $A$ в $i$-й ячейке, перенесем на ленту $B$ в ячейку с номером $2 i-1$, если $\alpha_{i}=0$, или в ячейку с номером $2 i$ если $\alpha_{i}=1$. Для этого потребуется $2 n$ шагов. После этого перенесем элементы списка назад на ленту $A$. Перемещая ленты $A$ и $B$ вправо, перенесем на ленту $A$ элементы, находящиеся в ячейках ленты $B$ с четными номерами. Для этого потребуется $2 n$ шагов. Затем, перемещая ленту $B$ в обратном направлении и продолжая перемещать ленту $A$ вправо, перенесем на ленту $A$ элементы, находящиеся на ленте $B$ в ячейках с нечетными номерами. Для этого так же потребуется $2 n$ шагов. Легко видеть, что общее время не превосходит $6 n$. Лемма доказана.

Теорема 1. Пусть $S$ - произвольная булева схема с п входами. Тогда для машины Тьюринга $M$ найдется такая программа $P$, что машина $M$ под управлением этой программы моделирует работу схемы $S$ и время работы $T(P)$ машины $M$ на программе $P$ при $L(S) \geqslant n$ и $n \rightarrow \infty$ удовлетворяет соотночению

$$
T(P)=O\left(L(S) \log _{2} L(S)\right) .
$$

Доказательство. Без ограничения общности будем полагать, что сложность моделируемой схемы $S$ является степенью двойки и равна $2^{m}$.

Очевидно, что моделирование одноэлементной схемы потребует не более четырех шагов. В качестве примера рассмотрим одноэлементную схему $S$, которая вычисляет трехкомпонентную вектор-функцию $(x, y, f(x, y))$. Допустим, что ее аргументы $x$ и $y$ перед началом работы находятся на ленте $A$ в двух соседних ячейках, левая из которых является активной ячейкой этой ленты. Тогда моделирование схемы $S$ можно провести следующим образом. На первом шаге автомат $Q$ запоминает символ $x$ из первой ячейки ленты $A$ и лента $A$ сдвигается влево. На втором шаге автомат $Q$ запоминает символ у из второй ячейки ленты $A$, и лента $A$ сдвигается вправо. На третьем шаге лента $A$ сдвигается вправо. На четвертом шаге в активную ячейку ленты $A$ помещается значение $f(x, y)$. В результате в трех соседних ячейках ленты $A$ записаны (неупорядоченно) значения векторфункции $(x, y, f(x, y))$, и самая левая непустая ячейка ленты $A$ является активной.

Допустим, что любая булева схема, состоящая из $2^{k}$ элементов, где $1 \leqslant k<m$, может быть промоделирована на машине Тьюринга за время $T\left(2^{k}\right) \leqslant 27 k 2^{k}$ и при этом будут выполнены следующие условия.

(a) В начальный момент времени входные данные, не более $2 \cdot 2^{k}$ символов, последовательно расположены на ленте $A$ в произвольном порядке. На ленте $A$ активной является самая левая непустая ячейка. Лента $B$ пуста. 
(b) Во время работы машины ни одна из ячеек, расположенных на ленте $A$ правее ячеек с входными данными, не является активной.

(c) После окончания работы машины результаты вычислений, не более $3 \cdot 2^{k}$ символов (аргументы схемы и вычисленные значения), последовательно расположены на ленте $A$. На ленте $A$ активной является самая левая непустая ячейка. Лента $B$ пуста.

Основываясь на сделанном предположении, опишем конструкцию программы $P_{m}$, моделирующей схему из $2^{m}$ элементов с $p \leqslant 2 \cdot 2^{m}$ входами и $q \leqslant 3 \cdot 2^{m}$ выходами. Команды программы $P_{m}$ разобьем на следующие четыре последовательных блока.

(1) Команды первого блока переупорядочивают входные данные на ленте $A$ так, чтобы данные, используемые первыми $2^{m-1}$ элементами схемы, были расположены левее данных, не используемых этими элементами. Из леммы 1 следует, что для этого потребуется не более $6 \cdot 2^{m+1}$ шагов. После выполнения команд первого блока на ленте $A$ активной является самая левая непустая ячейка.

(2) Команды второго блока выполняют вычисления, соответствующие первым $2^{m-1}$ элементам схемы, и удовлетворяют условиям $(a)-(c)$. В соответствии с предположением индукции для этого потребуется не более $T\left(2^{m-1}\right)$ шагов.

(3) Команды третьего блока переупорядочивают данные на ленте $A$ так, чтобы данные, используемые последними $2^{m-1}$ элементами схемы, были расположены левее данных, не используемых этими элементами. После выполнения команд второго блока на ленте $A$ может оказаться не более $3 \cdot 2^{m-1}$ значений, не являющихся аргументами последних $2^{m-1}$ элементов схемы. Поэтому в соответствии с леммой 1 для перемещения аргументов этих элементов потребуется не более $6\left(3 \cdot 2^{m-1}+2 \cdot 2^{m-1}\right)$ шагов.

(4) Команды четвертого блока выполняют вычисления, соответствующие последним $2^{m-1}$ элементам схемы, и удовлетворяют условиям $(a)-(c)$. В соответствии с предположением индукции для этого потребуется не более $T\left(2^{m-1}\right)$ шагов.

Индукцией по $m$ покажем, что время $T\left(2^{m}\right)$ работы программы $P_{m}$ удовлетворяет неравенству

$$
T\left(2^{m}\right) \leqslant 27 m 2^{m}
$$

При $m=1$ это неравенство очевидно, так как моделирование одноэлементной схемы выполняется не более чем за четыре шага. Допустим, что $T\left(2^{m-1}\right) \leqslant 27(m-1) 2^{m-1}$ при $m \geqslant 2$. Тогда из 1)-4) следует, что

$$
T\left(2^{m}\right) \leqslant 2 T\left(2^{m-1}\right)+54 \cdot 2^{m-1} \leqslant 2 \cdot 27(m-1) 2^{m-1}+54 \cdot 2^{m-1}=27 m 2^{m} .
$$

Теорема доказана.

4. Рассмотрим булевы схемы специального вида, схемы с ограниченным числом внутренних переменных. Булевой схемой $S$ с $n$ входами над множеством независимых переменных $\left\{x_{1}, \ldots, x_{n}\right\}$ и с $m$ внутренними переменными $\left\{y_{1}, \ldots, y_{m}\right\}$ будем называть последовательность присваиваний

$$
c_{i}=f_{i}\left(a_{i}, b_{i}\right), \quad i=1,2, \ldots, k,
$$


где $f_{i}$ - двухместная булева функщия, $c_{i} \in\left\{y_{1}, \ldots, y_{m}\right\}, i=1,2, \ldots, k$ и $a_{i}, b_{i} \in$ $\left\{x_{1}, \ldots, x_{n}, y_{1}, \ldots, y_{m}\right\}$. Равенства (2) называются элементами схемы, а их число - сложностью схемы. Как и ранее, сложность схемы $S$ обозначается через $L(S)$. С точки зрения сложности вычислений схемы с ограниченным числом внутренних переменных слабее обычных схем, так как в обычных схемах нет ограничений на число одновременно запоминаемых промежуточных результатов, а в схемах с $m$ внутренними переменными их число ограничено величиной $m$. Отражением этого факта является меньшее время моделирования схем с ограниченным числом внутренних переменных по сравнению со временем моделирования обычных схем равной сложности.

Теорема 2. Пусть $S$ - произвольная булева схема с $n$ входами и $m, m \geqslant n$, внутренними переменными. Тогда для машины Тьюринга $M$ найдется такая программа $P$, что машина $M$ под управлением этой программы моделирует работу схемы $S$ и время работы $T(P)$ машины $M$ на программе $P$ при $L(S) \geqslant n$ и $n \rightarrow \infty$ удовлетворяет соотношению

$$
T(P)=O\left(L(S) \log _{2} m\right) .
$$

Доказательство. Пусть $S$ - произвольная схема с $m$ внутренними переменными. Схему $S$ разобьем на $\lceil L(S) / m\rceil$ подсхем $S_{i}$, каждая из которых, кроме быть может последней, состоит из $m$ последовательных элементов схемы. Каждую такую подсхему будем рассматривать как самостоятельную схему с $m+n$ входами и $m$ выходами. Из предыдущей теоремы следует, что время моделирования каждой такой схемы (при условии $m \geqslant n$ ) по порядку не превосходит $m \log _{2} m$. Пусть $P_{i}$ - программа, моделирующая подсхему $S_{i}$. Нетрудно видеть, что программа $P$, состоящая в последовательном выполнении программ $P_{1}, P_{2}, \ldots, P_{\lceil L(S) / m\rceil}$, будет моделировать схему $S$. Поэтому

$$
T(P)=\sum_{i=1}^{\lceil L(S) / m\rceil} T\left(P_{i}\right)=\lceil L(S) / m\rceil O\left(m \log _{2} m\right)=O\left(L(S) \log _{2} m\right) .
$$

Теорема доказана.

5. Покажем, что с точностью до постоянного множителя оценки теорем 1, 2 точны. Для этого достаточно указать такие схему $S$ и схему с $m$ внутренними переменными $S^{\prime}$, что время вычисления на машине Тьюринга реализуемых этими схемами функщий с точностью до постоянного множителя не меньше, чем $L(S) \log _{2} L(S)$ и $L\left(S^{\prime}\right) \log _{2} m$, соответственно.

Рассмотрим множества $F(L, n)$ и $F(L, m, n)$, первое из которых состоит из всех $n$ местных булевых функций, сложность вычисления которых схемами не превосходит $L, a$ второе - из всех $n$-местных булевых функций, сложность вычисления которых схемами с $m$ внутренними переменными не превосходит $L$. Нетрудно показать (например, используя результаты из [10]), что при $L \geqslant m \geqslant n$ множества $F(L, n)$ и $F(L, m, n)$ содержат не менее $L^{c L}$ и $m^{c L}$ функций, где $c$ - некоторая постоянная (можно показать, что при $L / n \rightarrow \infty$ постоянная с стремится к единице). Поэтому среди функций из $F(L, n)$ и $F(L, m, n)$ обязательно найдутся такие функции $f \in F(L, n)$ и $g \in F(L, m, n)$, длины минимальных программ которых по порядку не меньше величин $L \log _{2} L$ и $L \log _{2} m$, соответственно. Легко видеть, что если это не так, то число различных программ (число программ длины $T$ не больше, чем $k^{T}$, где $k$ - постоянная) будет меньше числа функций.

Пусть $S_{f}$ - минимальная схема функции $f$ и $S_{g}$ - минимальная схема (среди всех схем с $m$ внутренними переменными) функции $g$. Так как время работы машины Тьюринга не 
меньше длины программы, легко видеть, что с точность до постоянного множителя время моделирования схем $S_{f}$ и $S_{g}$ не меньше, чем $L \log _{2} L$ и $L \log _{2} m$, соответственно.

6. В заключение отметим, что утверждения теорем 1 и 2 остаются справедливыми для машины Тьюринга с двумя лентами и для машины Тьюринга с одним магазином и одной лентой. Доказательства для этих машин аналогичны приведенным выше, но более громоздки.

\section{Список литературы}

1. Ахо А., Хопкрофт Дж., Ульман Дж., Построепие и апализ вычислительных алгоритмов. Мир, Москва, 1979.

2. Мальцев А. И., Алгоритмы и рекурсивные фуикции. Наука, Москва, 1986.

3. Сэвидж Д. Э., Сложность вычислений. Мир, Москва, 1998.

4. Хенни Ф. К., Стирнз Р. Е., Моделирование многоленточной машины Тьюринга на двуленточной. В кн.: Проблемы математической логики. Мир, Москва, 1970, с. 194-212.

5. Хенни Ф. К., Вычисления на машинах Тьюринга со входом. В кн.: Проблемы математической логики. Мир, Москва, 1970, с. 249-270.

6. Штосс Г. Й., Двуленточное моделирование машин Тьюринга. В кн.: Сложность вычислений и алгоритмов. Мир, Москва, 1974, с. 199-212.

7. Штосс Г. Й., $k$-ленточное моделирование $k$-головочных машин Тьюринга. В кн.: Сложность вычислений и алгоритмов. Мир, Москва, 1974, с. 190-198.

8. Schnorr C. P., The network complexity and the Turing machine complexity of finite functions. Acta Inform. (1976) 7, 95-107.

9. Чашкин А. В., Моделирование схем из функциональных элементов машинами Тьюринга. Дискретный анализ и исследование операчий (1999) 6, №3, 42-70.

10. Лупанов О. Б., Об одном подходе к синтезу управляющих систем - принципе локального кодирования. Пробл. киберн. (1965) 14, 31-110.

Статья поступила 21.04.2003. 